\title{
Non-Squamous Cell Carcinomas of the Larynx
}

\author{
Horakova Zuzana ${ }^{1}$, Gál Bretislav, Vesely Karel², Pazourkova ${ }^{2}{ }^{2}$, Pavla Urbanková ${ }^{1}$ and Szturz Petr ${ }^{3}$ \\ ${ }^{1}$ Department of Otorhinolaryngology and Head and Neck Surgery, St. Anne's University Hospital and Faculty of Medicine, Masaryk \\ University Brno, Czech Republic \\ ${ }^{2}$ Department of Pathology, St. Anne's University Hospital and Faculty of Medicine, Masaryk University Brno, Czech Republic \\ ${ }^{3}$ Department of Internal Medicine, Hematology and Oncology, University Hospital Brno and School of Medicine, Masaryk University, \\ Brno, Czech Republic
}

\section{Article Info

\author{
*Corresponding author: \\ Gál Bretislav \\ Department of Otorhinolaryngology and \\ Head and Neck Surgery, \\ St. Anne's University Hospital and Faculty \\ of Medicine \\ Masaryk University Brno \\ Czech Republic \\ Tel: +430543182923 \\ E-mail: b.gal@fnusa.cz after ph.num
}

Received: November 26, 2017

Accepted: April 3, 2018

Published: April 10, 2018

Citation: Horakova Z, Bretislav G, Karel V, Pazourkova M, Urbanková P, Petr S. NonSquamous Cell Carcinomas of the Larynx. Madridge J Otorhinolaryngol. 2018; 3(1): 41-46.

doi: $10.18689 / \mathrm{mjol}-1000108$

Copyright: ( 2018 The Author(s). This work is licensed under a Creative Commons Attribution 4.0 International License, which permits unrestricted use, distribution, and reproduction in any medium, provided the original work is properly cited.

Published by Madridge Publishers

\begin{abstract}
Malignant laryngeal tumours are frequent pathologies in the head and neck region. Histologically, the vast majority are represented by squamous cell carcinomas (SCC).

Here in, we retrospectively reviewed patients treated in a single hospital centre over the last 13 years. Only 4 out of the total of 654 cases were diagnosed with a different histology than SCC (1 chondrosarcoma, 2 neuroendocrine and 1 myoepithelial carcinomas).

They are presented as a case series.
\end{abstract}

Keywords: Laryngeal malignancy, non-squamous cell carcinoma, chondrosarcoma, myoepithelial carcinoma, neuroendocrine carcinoma

\section{Introduction}

Based on data from the Czech National Cancer Registry, laryngeal cancer is an ENT (ear nose throat) malignancy with an incidence of about 515-564 new cases per year in the Czech population over the past three decades, with a mortality of 247-307 and a male preponderance of about 1: 9 . The vast majority of cases comprise squamous cell carcinomas (SCCs) [1]. In this retrospective study we focused on cases of non-SCC histologicy treated in our centre over the past 13 years (2001-2013). Only 4 out of 654 patients (i.e. less than 1\%), here presented as a case series, were found to be of non-SCC histology ( 1 chondrosarcoma, 2 neuroendocrine and 1 myoepithelial carcinomas).

\section{Case report No. 1: Chondrosarcoma}

A 57-year-old female with a history of smoking was followed-up for hoarseness at our ENT outpatient centre for several years. A sudden onset of a progressively developing dyspnoea over the preceding days brought her urgently to our clinic. An urgent tracheotomy was performed, and rigid laryngoscopy revealed an infiltrate of the inner surface of the thyroid cartilage under an intact mucosa resulting in bulging of the plica ventricularis and vocalis. This a typical submucosal infiltrate of supraglottis reduced the glottis to $1-2 \mathrm{~mm}$ at the posterior commissure. Computed tomography (CT) showed an encapsulated, spherical, slightly enhanced mass deforming the laryngeal skeleton and crushing the cricoid cartilage (Figure 1). 


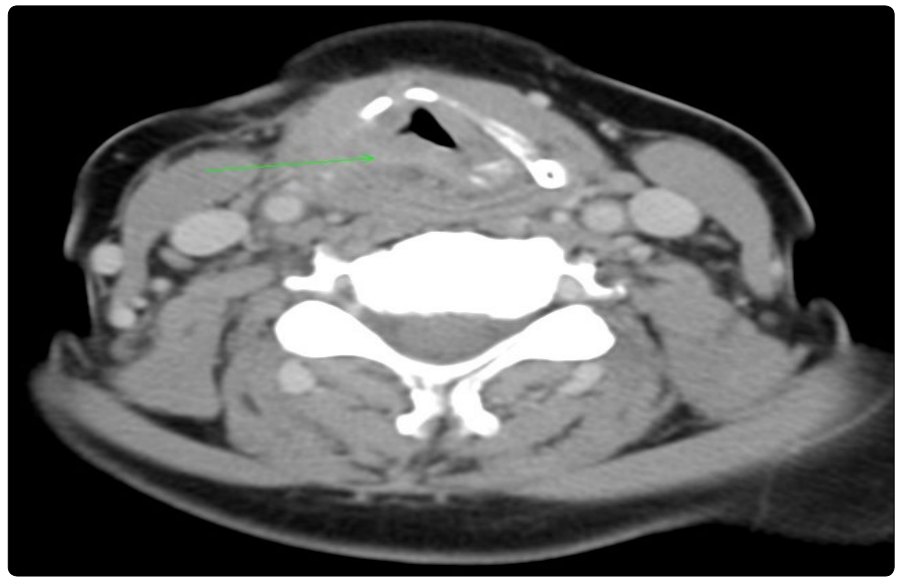

Figure 1a. Laryngeal and pharyngeal entry asymmetry, crushed upper cricoid margin, soft tissues, maximum at the arytenoid level, pushing the glottis to the right and slightly bulging subglottically

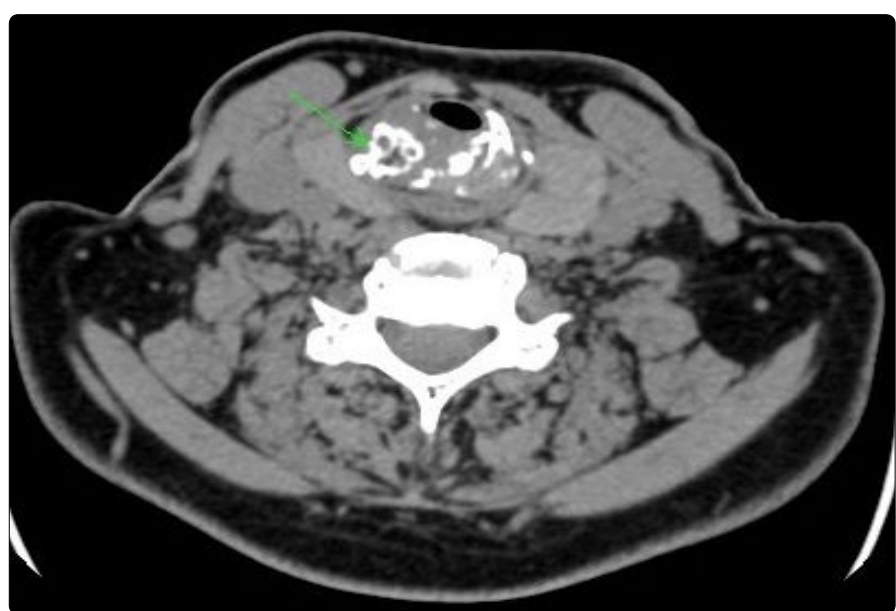

Figure 1b. Subglottic calcified recurring tumour destructing the thyroid and cricoid cartilages.

Surgical therapy was suggested; a conservative external approach was preferred due to the cystoid bordered character, unknown histology and no suspicion of an aggressive behaviour. Through a resection of the upper corner of the thyroid cartilage, a tough, non-smooth spherical tumour of cartilaginous character, about 2 by 2 by $2.5 \mathrm{~cm}$, originating from the upper ridge of cricoid cartilage was reached. It was then per partes submucosally removed under endoscopic control. Histopathology confirmed a low-grade chondrosarcoma (G1) - lobulated cartilaginous tumour with presence of binucleated neoplastic cells and focal regressive changes. Immunohistochemistry (IHC): p53 - focally positive, proliferative antigen Ki-67 - focally increased (Figure 2a).

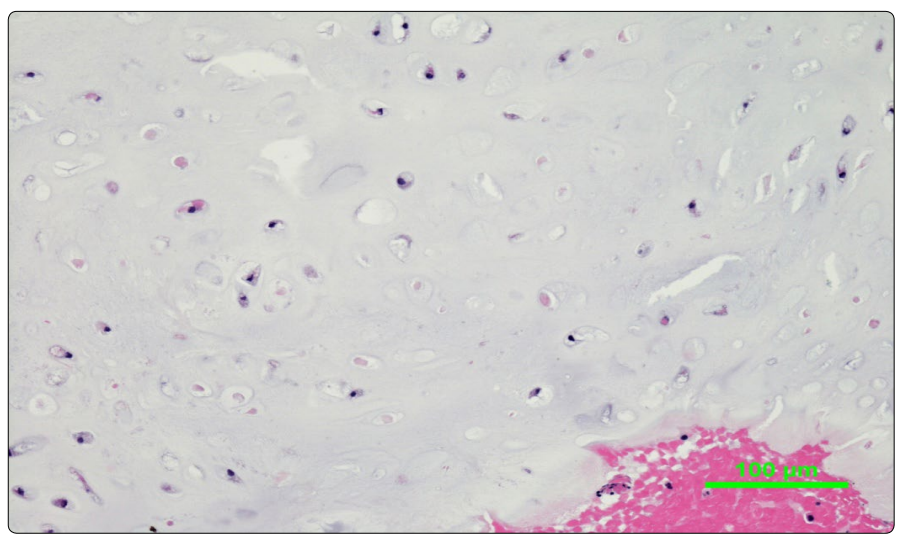

Figure 2a. G1 with low cellularity and neoplastic chondrocytes set in abundant hyaline intercellular matrix $(H \& E, x 200)$.

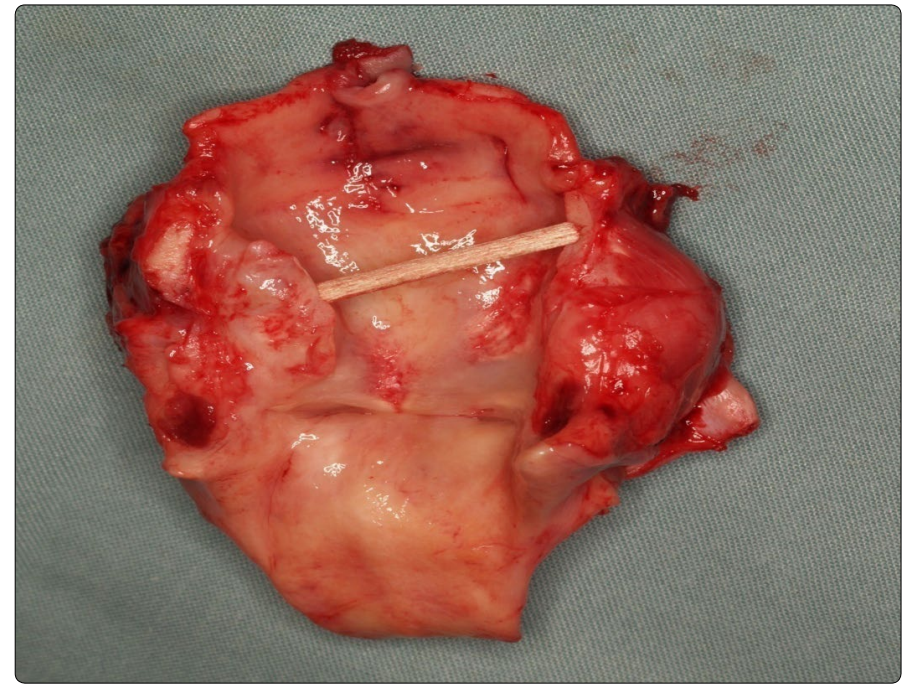

Figure 2b. Subglottic recurring chondrosarcoma - resected larynx

Assuming non-aggressive biological behaviour, radiotherapy was not indicated. Postoperatively, a noticeable arytenoid swelling persisted reducing the glottis to $2 \mathrm{~mm}$ and excluding the possibility of decannulation. After six months, laryngoscopy confirmed persistence of laryngeal hemiparesis with a smooth elastic bulging of the ventricular plica caused by submucosal mucous retention. Under a regular endoscopic follow-up, a new pathology was diagnosed: a subglottic, tough, submucous bulging. A CT scan (Figure $1 \mathrm{~b}$ ) raised suspicion of a tumour recurrence. A salvage total laryngectomy was performed. Histopathology confirmed a recurrence of a low-grade chondrosarcoma (G1), about 3 by 3 by $3.5 \mathrm{~cm}$ in diameter (Figure 2b), originating from the lower ridge of the cricoid cartilage. Currently, after 2 years of follow-up, the patient shows no signs of disease but is obviously tracheostomy dependent (both overall and disease-free survivals are 96 months).

\section{Case report No. 2, Neuroendocrine Carcinoma}

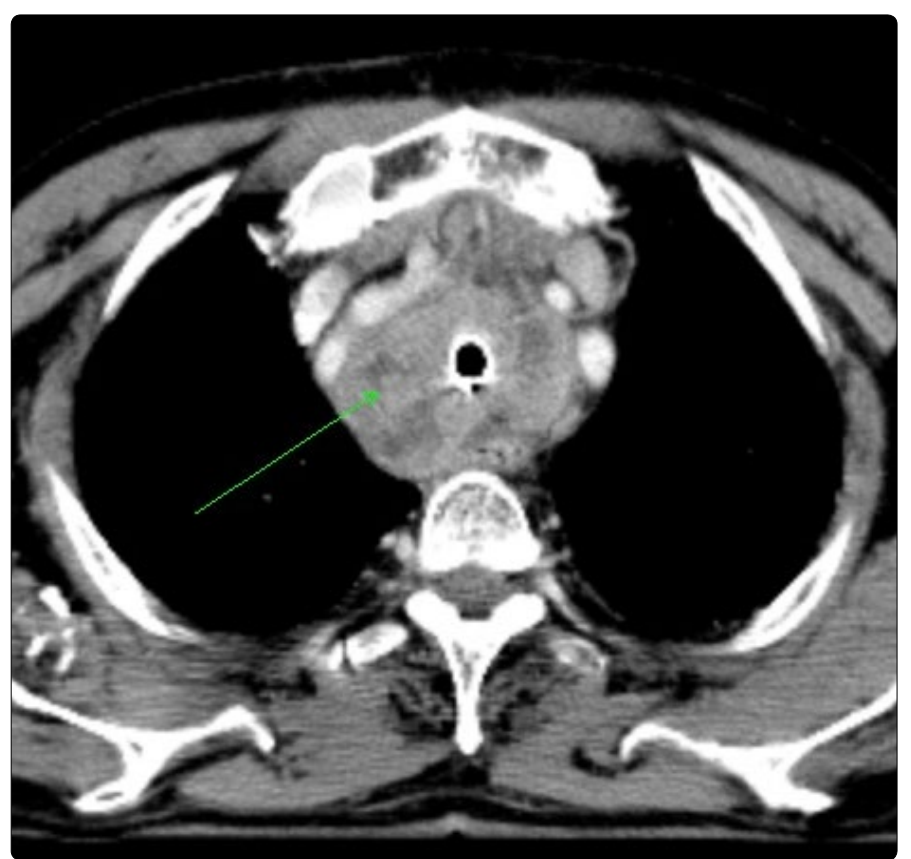

Figure 3. Packets of partly necrotic lymph nodes of the upper mediastinum surrounding the trachea and vessels. 


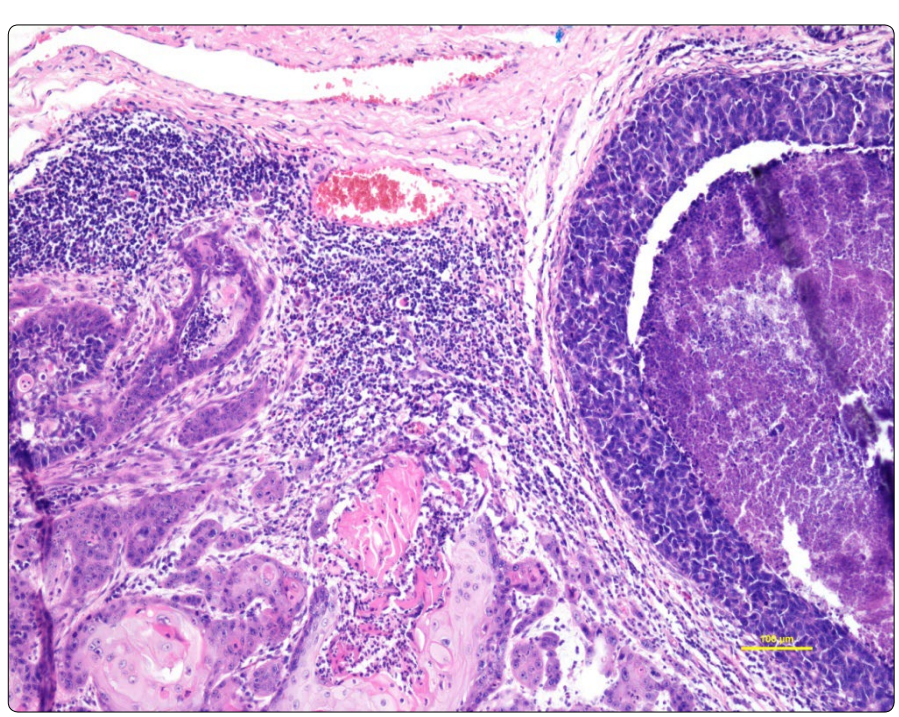

Figure 4. Squamous cell carcinoma (left) and large cell neuroendocrine carcinoma (right), $\left(H \& E_{1} \times 100\right)$

A 76-year-old male, a non-smoker since the age of 40 , complained of hoarseness persisting for two months after a respiratory infection. An examination followed by endoscopy showed a supraglottic tumour (grainy, exophytic, involving the anterior glottic commissure) with laryngeal hemiparesis. The pathological finding of a biopsy specimen revealed lowgrade SCC. A CT scan (Figure 3) indicated a possibly malignant tumour crushing the cartilaginous skeleton (CT4 cNO). A total laryngectomy was carried out with histology results surprisingly corresponding with a combination of SCC and giant cell neuroendocrine carcinoma 2 by $2.5 \mathrm{~cm}$ in diameter which was also confirmed by IHC staining (Figure 4). Structures of keratinizing spinocellular carcinoma with papillary growth pattern were present in the superficial parts of the tumour, with transition into invasive structures of undifferentiated carcinoma (G4). The deeper invasive infiltrate was formed by smaller cells with a high nuclearcytoplasmic (N/C) ratio and numerous mitotic Figureures. Occasional rosette formation and focally admixed tingiblebody macrophages giving rise to starry-sky appearance were also seen. IHC staining results showed strong expression of CK5 in the superficial areas; CK7 expression and coexpression of neuroendocrine markers (chromogranin and CD56) in deeper parts of the tumour with morphological features corresponding to the large cell neuroendocrine carcinoma.

Resection was performed with clear margins (pT4), followed by radiotherapy. At ten months, a CT examination showed a recurrence in the mediastinal lymph nodes which indicated the patient to receive a second series of palliative radiotherapy. In two months, the patient died of respiratory failure at home, before the effect of radiotherapy could have been assessed (overall survival 13 months, disease-free survival 10 months).

\section{Case report No. 3, Neuroendocrine Carcinoma}

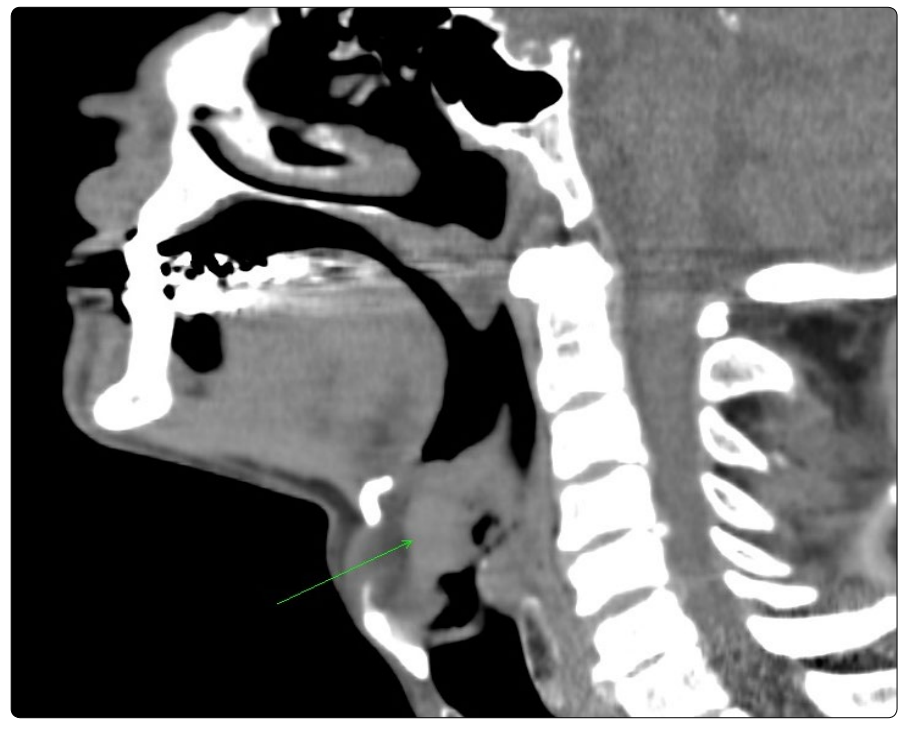

Figure 5. Supraglottic tumour right, centrally necrotic, expanding to the preepiglottic space, affecting the aryepiglottic plica, arytenoid and epiglottis, filling in the right piriform sinus and leading to compression of the right vallecula, without evident thyroid cartilage destruction.

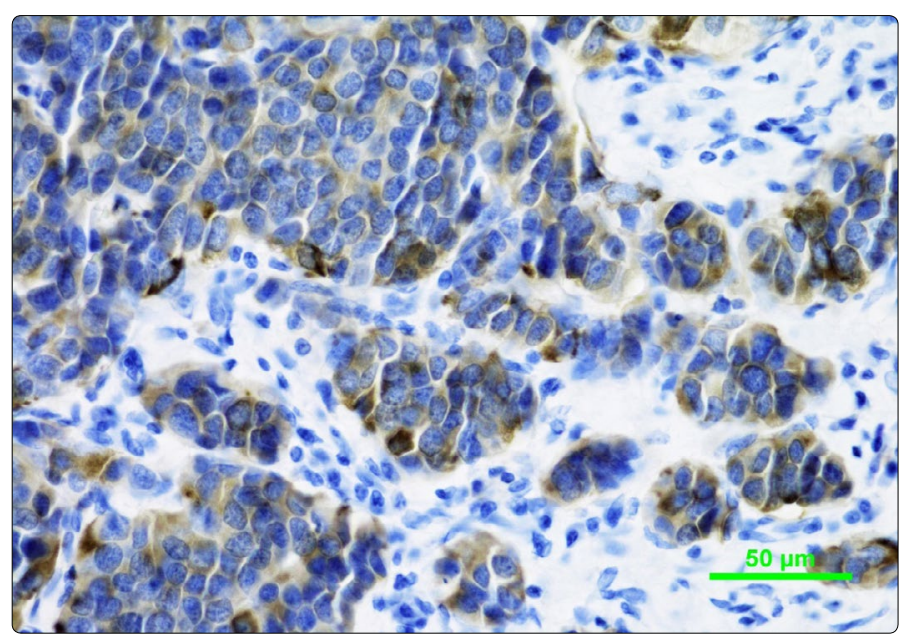

Figure 6. Confirmation of neuroendocrine differentiation by immunohistochemical staining: chromogranin A positivity in moderately differentiated neuroendocrine carcinoma G2. (x400).

A 64-year-old male, a non-smoker for the last 6 years, was complaining of dysphagia and odynophagia for 4 months. An endoscopy was performed. The finding in the larynx was unclear (epiglottis and ventricular fold mucosa surface covered with whitish necrotic tissue, hemilaryngeal paresis), while a CT scan revealed an extensive infiltrate involving the thyroid and epiglottic cartilages (Figure 5), expanding into the preepiglottic space (cT4 cNO). Histology indicated SCC, and the patient underwent a total laryngectomy with bilateral elective neck dissection. Postsurgical histology of the ulcerated tumour, 2 by $2.5 \mathrm{~cm}$ in diameter, invading through the epiglottic cartilage to preepiglottic space surprisingly revealed a moderately differentiated neuroendocrine carcinoma (G2). Metastatic infiltration was detected in three lymph nodes. The neoplastic infiltrate consisted of irregular solid clusters and trabecular formations, set in a fibrous stroma (Figure 6). The neoplastic cells were moderate to large in size, with vesicular nuclei and focally prominent nucleoli. Focal regressive changes, confluent 
at some areas, and lymphangiovascular invasion were also present. Mitotic activity was high, up to 16 mitotic Figure in 10 high-power fields (HPF). IHC staining: chromogranin, synaptophysin and CD56 - positive, CK5 - negative.

Resection was carried out with clear margins, metastases were identified in 3 lymph nodes (pT4 N2b). Radiotherapy followed. At 16 months, the disease recurred with metastases to multiple skin sites (arms, thighs, buttocks), both testes and the inguinal lymph nodes. Skin metastasectomies, bilateral orchiectomy and palliative irradiation were carried out. At 22 months, diplopia developed due to an orbital metastasis, which was excised via lower orbitotomy and irradiated. At 12 months, the patient died due to rapid progression of multiple lung, skin and orbital metastases (overall survival 34 months, disease-free survival 16 months).

\section{Case report No. 4: Myoepithelial Carcinoma}

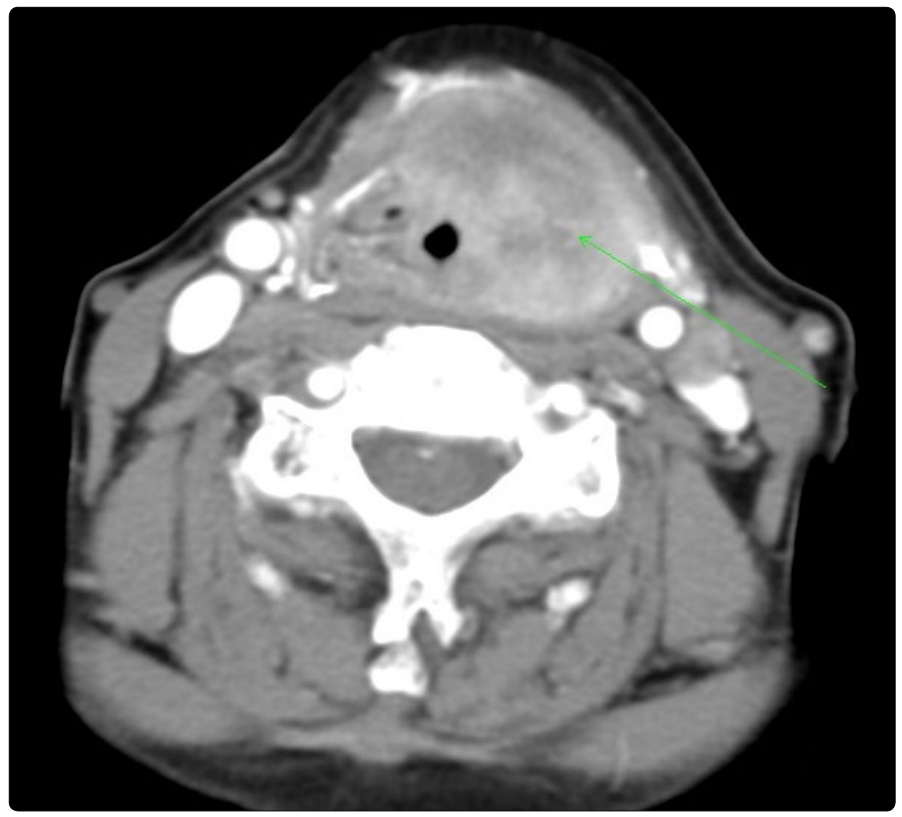

Figure 7. Supraglottic tumour left, expanding to the pre epiglottic space, crossing the midline to the left, crushing the upper thyroid margin and resulting in ossification of the cartilage remnant, completely filling in the left piriform sinus.

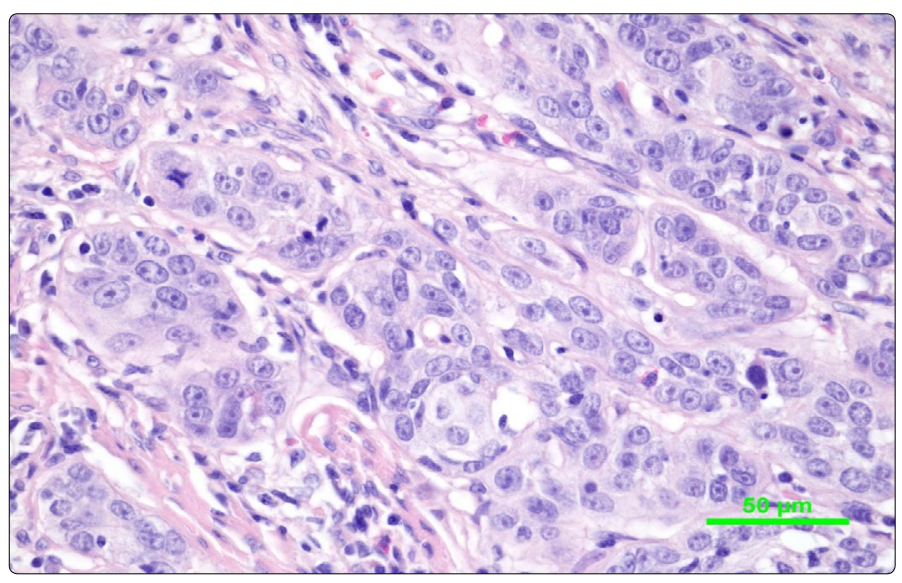

Figure 8. Neoplastic cells with round to ovoid nuclei, prominent nucleoli and small amount of eosinophilic up to amphophilic cytoplasm, proliferating in trabeculae with formation of abortive glandular lumina. (H\&E, x400).
An 82-year-old female, with out a history of smoking, suffered from dysphagia for two months, accompanied by a rapidly progressive dyspnoea later on. Clinical examination immediately revealed a possible tumour at the laryngeal entrance. A CT imaging confirmed a tumour, 4 by $3 \mathrm{~cm}$ in diameter, involving the supraglottis, expanding through the epiglottic and thyroid cartilages and invading into the preepiglottic space (Figure 7). An urgent tracheotomy, followed by endoscopy, was performed (spheric purple solid tumour, 2.5 by 2.5 by $3 \mathrm{~cm}$ affecting the glossoepiglottic fossa, base of the epiglottis, aryepiglottic plica, arytenoid and ventricular cords, extending towards hypopharynx). Histology revealed myoepithelial carcinoma of the salivary glands. Due to the age and performance status of the patient, total laryngectomy (cT4 cN0) without neck dissection was performed finding a hard, whitish, homogeneous tumour submucosaly encircling the thyroid cartilage, 3 by 3.5 by $4 \mathrm{~cm}$ in diameter. The parapharyngeal spaces were clear without lymphadenopathy.

Histology confirmed a clear cell variant of myoepithelial carcinoma (Figure 8), intermediate to high-grade (G2-3), with formations of spindle and epithelioid cells and admixture of clear cell component. The epithelial structures showed mixture of solid, fascicular and trabecular growth pattern. The stroma was predominantly desmoplastic and focally myxoid. The neoplastic cell population showed mild to significant cytonuclear atypia. Mitotic Figureures were numerous. IHC staining: CK14+, p63+, calponin+, AE1/AE3+, GFAP-, Ki-67 focally exceeding $30 \%$.

Early on, during the scheduled $\mathrm{CT}$, prior to the radiotherapy, a suspicious metastasis appeared in the lymph node situated laterally to the hypopharynx. Radiotherapy of the hypopharynx and regional lymphatic system followed.

After 10 months of follow-up, a salvage neck dissection of the contralateral submandibular area was performed (hypoechogenic infiltrate on ultrasound; 2.2 by $1.6 \mathrm{~cm}$ ), and a significantly larger mass (4 by $3.5 \mathrm{~cm}$ ), fixed to the $\mathrm{m}$. digastricus and bifurcation of a. carotis, in filtrating the wall of v. jugularis interna, $\mathrm{n}$. XII and $\mathrm{m}$. sternocleidomastoideus was found. Histology confirmed the recurrence of myoepithelial carcinoma (G3) with no lymph node involvement (0/8). Subsequently, a second series of palliative radiotherapy was delivered to the contralateral side of neck. Afterwards the patient was without evidence of tumour for 10 months (overall survival 26 months, disease-free survival 10 months). However, the patient suddenly died while under home care. This was most likely due to recurrent respiratory infections and the generally poor health status.

\section{Discussion}

Over $95 \%$ of all laryngeal malignancies are SCC. Non-SCC malignancies comprise a broad spectrum of histology. Due to the low incidence and pathological variability, Prediction of their biological behaviour and prognosis and standardizing treatment recommendations is complicated. The majority of metastases are found in the supraglottic area. Histologically, 
they include neuroendocrine, adenosquamous, adenoid cystic or mucoepidermoid carcinomas, lymphomas or sarcomas, while others emerge very rarely. Despite the small number of non-SCC cases, this retrospective review reflects data of the most extensive retrospective comparative study, which was based on the US national cancer database [2].

\section{Chondrosarcoma}

Chondrosarcoma, the most common type of laryngeal sarcoma, represents less than $0.5-1 \%$ of all laryngeal malignancies; about 200 cases have been published so far [3]. Etiopathogenesis is unclear, hypotheses suggest previous injury, ossification abnormalities (usually at the muscle insertion), chronic inflammation, ischemic changes, metabolic abnormalities after previous surgery or radiotherapy as contributing factors. They are closely related to chondromas, both synchronic and metachronic. They typically affect older men (50-70 years), which corresponds to the age of hyaline cartilage ossification. Tumours most frequently originate from the cricoid cartilage $(75 \%)$, mainly its posterior lamina, thyroid (20\%) and arytenoid (3\%), presenting with a slowly progressive dyspnoea, dysphagia, cough and stridor. Endoscopy usually reveals submucosal infiltrate, a tumorous mass on $\mathrm{CT}$, often with focuses of calcification, with minimal contrast uptake, on magnetic resonance imaging $\mathrm{T} 2$ hypersignal and T1 hyposignal. Prognosis is generally favourable. In the case described above, the tumour grew under intact mucosa, only indirect signs (disFigureuration and lumen reduction) of the tumour were evident under endoscopy; $\mathrm{CT}$ imaging showed a well-bordered infiltrate, the slow growth of which was indicated by skeletal deformation without infiltration or destruction. They are histologically formed by immature chondrocytes with nuclear abnormalities, more frequently of higher grading ( $40-64 \%$ G1, 28-49\% G2 and 5-8\% G3) as seen in our patient. Surgery is usually the first choice of treatment, endoscopic or conservative external access (removal of the tumour with safe margins but with an emphasis on preserving laryngeal function). Total laryngectomy is indicated only exceptionally in relapses. Radiotherapy is reserved for unresectable tumours or after incomplete excision. Chemotherapy is of no clinical significance. Local recurrence and metastases are rare, usually following incomplete excision or a diagnosis of low-grade histology. Recurrence does not significantly affect prognosis. Salvage laryngectomy results are comparable to those of the larynx-preserving procedures, thus allowing emphasis on the most conservative surgical procedures with respect to the post-treatment quality of life preservation. The overall 5 -year survival rate is estimated at about 90-97\% [3-5]. In accordance with these assumptions, the patient was successfully treated with a conservative surgical approach without radiotherapy and during 5 years of follow-up she was without evidence of tumour. Unfortunately, due to the considerable extent of the tumour involving the glottis and vocal cords, she was tracheostomy dependent but still benefiting from a good voice quality. The recurrence was later managed by salvage total laryngectomy. Currently, there is no evidence of tumour recurrence.

\section{Neuroendocrine carcinomas}

Following SCC, neuroendocrine carcinomas are the second most frequently observed head and neck cancers. According to histological grading system, they are classified into poorly, moderately and well differentiated tumours (also known as small cell carcinomas, atypical and typical carcinoids). Grading is regarded as the principal independent prognostic factor [6].

Well differentiated carcinoma, where conservative surgery is recommended, has the lowest incidence (only 15 cases published in literature so far) and the best prognosis.

Poorly differentiated ones are the most aggressive frequently presenting with distant metastases and have very poor prognosis (5-year overall survival about 5\%). Chemoradiotherapy as first line treatment is suggested [7].

Moderately differentiated carcinoma is the most common variant, typically occurring in older men and smokers, presenting with odynophagia and dysphagia, while carcinoid syndrome and systemic symptoms are rare. Submucosal infiltrative growth and predilection for the supraglottic area are typical, which was confirmed in both our cases, where the final extent was significantly larger compared to the initial endoscopic assessment. Although this type of histological finding is typical (eosinophilic cells immunopositive to synaptophylin, chromogranin, keratin, calcitonin and CEA, frequent mitosis, cellular pleomorphism and necrosis), assessment is problematic, especially in limited tissue samples. In our patient (case No. 2), the first biopsy indicated SCC. Surprisingly, the definitive post-surgical results revealed a neuroendocrine carcinoma. In such cases, radical surgery including neck dissection followed by radiotherapy is recommended. In case of inoperabilty, primary radiotherapy is indicated. Despite of that, aggressive behaviour with rapid growth, early recurrence (locoregional and distant) and high mortality is to be expected; distant subcutaneous metastases are also typical as was the case with our patient. However, the problem of optimizing therapeutic recommendations remains, since only rare cases have been published (about 300 so far, usually as case reports) [8]. Despite aggressive multimodal treatment, both our patients experienced poor survival with early recurrence (mediastinal metastases in case No. 2, and subcutaneous metastases followed by orchitic and orbital ones in case No. 3), and rapid progression.

\section{Myoepithelial carcinoma}

The most common histologic variant of tumours originating from the small salivary glands of laryngeal mucosa are adenoid cystic (30-75\%) and mucoepidermoid carcinomas (15-35\%) whereas myoepithelial carcinoma is extremely rare (less than 1\%). To the best of our knowledge, only four cases have been reported so far [9-13]. Typical infiltrative growth with perineural and perivascular spread results in a late onset of symptoms in advanced stages, often revealing a considerably larger tumour after surgery than expected on endoscopy. Tumour size, cell proliferative activity and 
polymorphism are supposed to be the strongest prognostic factors. Radical surgery followed by radiotherapy should be performed. Generally, early relapse, both local and distant, with poor prognosis can be observed. All cases reported so far relapsed early and, despite salvage surgery, the patients died because of disease progression. Unfortunately, this also held true in our patient, where even multimodality treatment did not prevent the development of early locoregional recurrence.

All of the aforementioned non-SCC cases were supraglottic tumours with mostly submucous infiltrative growth. Probably owing to the late onset of non specific symptoms, they were diagnosed at an advanced stage (IV). Although the patients underwent combined radical treatment, their outcome, except for the patient with chondrosarcoma, was poor.

\section{Conclusion}

Laryngeal malignancy is a common type of cancer in ENT practice. From a histological point of view, over $95 \%$ of patients are diagnosed with SCC. In the remainder, a wide variety of other histological types can be identified. That said, all published studies on these rare cases suffered from a small number of patients, illustrating the histological and clinical variability of such tumours. This should be regarded as a challenge, attracting attention and maintaining awareness of both clinicians and pathologists. Nevertheless, standard therapeutic recommendations can still hardly be established. In our retrospective review of laryngeal malignancies covering the past 13 years we tried to address these issues finding non-SCC in less than $1 \%$ patients (4 out of 654 ).

\section{References}

1. Ladislav D, Jan M, Miroslav K, Jana K, Jan Z, Rostislav Y. Epidemiology of Malignant Tumours in the Czech Republic. 2007.

2. Lin $H$, Bhattacharyya $N$. Staging and survival analysis for nonsquamous cell carcinomas of the larynx. Laryngoscope. 2008; 118: 1003-1013. doi: 10.1097/MLG.0b013e3181671b3d

3. Thompson LDR, Gannon FH. Chondrosarcoma of the larynx. Am J Surg Pathol. 2002; 26: 836-851.

4. Nao EM, Bozec A, Vallicioni J, Poissonnet G, Riss JC, et al. Laryngeal chondrosarcoma: report of two cases. European Annals of Otorhinolaryngology, Head and Neck diseases. 2011; 128: 191-193.

5. Christiansen $\mathrm{H}$, Hermann RM, Pradler $\mathrm{O}$, Martin $\mathrm{A}$, Schmidberger $\mathrm{H}$, Adjuvant radiotherapy after surgical resection led to long term survival in multiple recurrent chondrosarcoma of the larynx. Case report. Clin Pract Rev. 2005; 3: 65-68.

6. Gillenwater A, Lewin J, Roberts D, El Nagfar A. Moderately differentiated neuroendocrine carcinoma (atypical carcinoid) of the larynx: A clinically aggressive tumor. Laryngoscope. 2005; 115: 1191-1195. doi: 10.1097/01. MLG.0000166179.40750.1B

7. Bapat U, Mac Kinnon NA, Spencer MG. Carcinoid tumours of the larynx Eur Arch Otorhinolaryngol. 2005; 262: 194-197.

8. Chung JH, Lee SS, Shim YS, Kim SY, Nam SY, et al. A study of moderately differentiated neuroendocrine carcinomas of the larynx and an examination of non- neoplastic larynx tissue for neuroendocrine cell. Laryngoscope. 2004; 114: 1264-1270. doi: 10.1097/00005537-20040700000023

9. Ibrahim R, Bird DJ, Skelet MW. Malignant myoepithelioma of the larynx with massive metastatic spread to the liver: an ultrastructural and immunocytochemical study. Ultrastruct Pathol. 1991; 15: 69-76.

10. Ganly I, Patel SG, Coleman M. Malignant minor salivary gland tumors of the larynx. Arch Otolaryngol Head Neck Surg. 2006; 132: 767-770. doi: 10.1001/archotol.132.7.767

11. Savera AT, Sloman A, Huvos AG, Kalimstra DS. Myoepithelial carcinoma of the salivary gland: a clinicopathologic study of 25 patients. Am J Surg Pathol. 2000; 24: 761-774.

12. Mao YJ, Luo XM, Zhou SH, Zheng ZJ. Calponin expression in laryngeal myoepithelial carcinoma and its prognostic implications: a case report and literature review. Journal int med research. 2010; 38: 711-719. Doi: $10.1177 / 147323001003800236$

13. Yu G, Qu G, Kong L, Pan X. Primary myoepithelial carcinoma of the larynx: case report and review of the literature. Patology- research and practice. 2011; 207: 127-130. doi: 10.1016/j.prp.2010.10.006 